\title{
Research on Periodic Motion Stability of Rotor-Bearing System with Dual-Unbalances
}

\author{
Chaofeng $\mathrm{Li}^{\mathrm{a}}$, Guangchao $\mathrm{Liu}^{\mathrm{b}}$, Qingliang $\mathrm{Li}^{\mathrm{c}}$ and Bangchun Wen ${ }^{\mathrm{d}}$ \\ School of Mechanical Engineering \& Automation, Northeastern University, Shenyang, China \\ achfli@mail.neu.edu.cn, 'lifeeenjoy@gmail.com, 'liqinliang@vip.qq.com, ${ }^{\mathrm{b}} \mathrm{bcwen1930@sina.com}$
}

Key words: rotor-bearing system, bifurcation, stability, dimension reduction, instability speed

\begin{abstract}
Multiple freedom degrees model of rotor-bearing system taking many factors into account is established, the Newmark- $\beta$ and shooting method are combined during the stability analysis of periodic motion in such system. The paper focused on the influence law of two eccentric phase difference on the instability speed of rotor-bearing system. The results have shown that the instability speed rises constantly with the eccentric phase difference angle increasing in small eccentricity system. When the two unbalance be in opposite direction, the system reached its maximum instability speed. However, the unstable bifurcation generates mutation phenomenon for large eccentricity system with the eccentric phase difference angle increasing. In summary, the larger initial phase angle can inhibit system instability partly. The conclusions have provided a theoretical reference for vibration control and stability design of the more complex rotor-bearing system.
\end{abstract}

\section{Introduction}

In recent years, many scholars have carried out a lot of research on nonlinear dynamic characteristic of the rotor-bearing system supported by oil-film. Brancati ${ }^{[1]}$ studied journal orbits and their stability for rigid unbalance rotor supported by cylindrical bearings. Kicinski ${ }^{[2]}$ gave a new method to determine the liner dynamic coefficients of the bearing, and studied nonlinear dynamics of multi-span rotor system with its finite element model, Feng ${ }^{[3]}$ used the transfer matrix method with the transient linear dynamic coefficients of the bearing for studying the stability problems of indeterminate multi-span shaft. $\mathrm{Jiao}^{[4]}$ analyzed dynamic characteristic of rotor-cylindrical bearing system with different oil-film force models including database method using the discrete model. In order to study dynamic characteristic of high-dimensional rotor systems, Zheng ${ }^{[5]}$ established a model of the high-dimensional rotor system and proposed a dimension reduction method for the analysis on periodic stability of the model. Jing ${ }^{[6,7]}$ established lower-dimensional finite element model with fixed-interface modal synthesis method, then carried out comparative analysis on dynamic characteristic of multi degree of freedom model and the discrete model; Wang ${ }^{[8]}$ established dynamics model of turbogenerator shaft elliptical bearing-rotor system with the finite element method, and studied its stability and bifurcation with different methods based on the dimension reduction. However, as the system stability and response caused by eccentric phase of turntable has been relatively little research, it is necessary to study this issue.

Based on the double-disc multiple degrees of freedom rotor-bearing system, the system stability and bifurcation behavior is studied in the eccentricity-speed, eccentric phase-speed parameters domain by the continuation-shooting algorithm for periodic solution of nonlinear non-autonomous system in this paper. The law of instability and of bifurcation of the system periodic motion is obtained and theoretical reference is provided for the vibration control, fault diagnosis and stability of the design of rotor-bearing system. 


\section{Dynamic model of the rotor-bearing system}

The research object in this paper is a simplified double-disc rotor-bearing system supported by oil-film. Its finite element model is shown as Fig. 1, which consists of two discs that are located about two thirds of the marked point and two cylindrical bearings and a shaft. The related parameters of rotor-bearing system as follows: both ends bearing diameter $\mathrm{D}=44 \mathrm{~mm}$, effective length are $\mathrm{Lr}=20 \mathrm{~mm}$; Rotor shaft radius $\mathrm{r}=18 \mathrm{~mm}$, length $\mathrm{L}=720 \mathrm{~mm}$, two disks diameter $\mathrm{Ro}=$ $160 \mathrm{~mm}$, the thickness $\mathrm{H}=20 \mathrm{~mm}$, bearing average bearing clearance $\mathrm{c}=0.18 \mathrm{~mm}$, two disks eccentric $\mathrm{b}=0.10 \mathrm{~mm}$, two disks eccentric initial phase $\varphi_{1}=\varphi_{2}=0$, phase difference $\Delta \varphi=0$, lubricating oil kinetic viscosity $\mu=0.018 \mathrm{~Pa} \cdot \mathrm{s}$. Eccentric initial phase means the angle between eccentric direction and the $\mathrm{x}$ axis. phase difference means the angle between the two Eccentric initial phase angles.

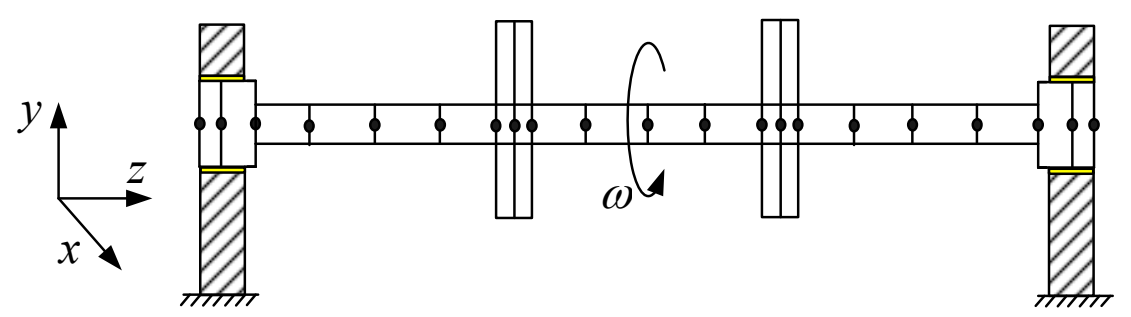

Fig. 1. Finite element model of the double eccentric rotor-bearing system supported by oil film

According to the particularity of the research object, the mathematical model is established with isoparametric beam element. Ignoring the axis translational and rotational effects of the rotor system, each node in each element has two translational degrees of freedom and two rotational degrees of freedom, 8 degrees of freedom totally, respectively represented by $q_{1} \sim q_{12}$. The motion differential equation of the shaft element including material internal resistance is as follows:

$$
\left(\boldsymbol{M}_{\mathrm{T}}^{\mathrm{e}}+\boldsymbol{M}_{\mathrm{R}}^{\mathrm{e}}\right) \ddot{\boldsymbol{q}}^{\mathrm{e}}+\left(\alpha \boldsymbol{M}^{\mathrm{e}}+\beta \boldsymbol{K}^{\mathrm{e}}+\Omega \boldsymbol{J}^{\mathrm{e}}\right) \dot{\boldsymbol{q}}^{\mathrm{e}}+\boldsymbol{K}^{\mathrm{e}} \boldsymbol{q}=\boldsymbol{Q}^{\mathrm{e}}
$$

where, mass matrix including translational mass matrix $\boldsymbol{M}_{T}^{e}$ and rotation mass matrix $\boldsymbol{M}_{R}^{e}, \boldsymbol{J}^{e}$ is the gyroscopic matrix, $\boldsymbol{K}^{e}$ is the element stiffness matrix, $\alpha, \beta$ is the material damping coefficient, $\boldsymbol{Q}^{e}$ is the vector sum of external force vector acting on the shaft.

The FE model of the rotor system is shown in Fig. 2, where each dot represents a node and each section represents an element. With the nonlinear oil- film force

vector at both ends added on the right of the motion equation, the motion equation of the system is as following:

$$
\ddot{\boldsymbol{M} \boldsymbol{X}}+\overline{\boldsymbol{C}} \dot{\bar{X}}+\overline{\boldsymbol{K} \boldsymbol{X}}=\boldsymbol{F}(\overline{\boldsymbol{X}}, \dot{\bar{X}})+\boldsymbol{R}(t)+\boldsymbol{G}
$$

where, $\quad \overline{\boldsymbol{C}}=(\boldsymbol{D}+\boldsymbol{J} \cdot \omega), \quad \overline{\boldsymbol{X}}=\left[q_{1}, q_{2}, \cdots, q_{n}\right]^{\mathrm{T}}, \quad(\mathrm{n}=17), \quad q_{i}=\left[x_{\mathrm{i}}, y_{\mathrm{i}}, \theta_{\mathrm{xi}}, \theta_{\mathrm{yi}}\right],(\mathrm{i}=1,2, \ldots, 20)$

$\overline{\boldsymbol{M}}$ is the mass matrix of the system; $\overline{\boldsymbol{C}}$ is the damping matrix of the system; $\boldsymbol{D}$ is the material damping matrix; $\boldsymbol{J}$ is the gyroscopic matrix; $\omega$ is the rotation speed; $\overline{\boldsymbol{K}}$ is the stiffness matrix; $\overline{\boldsymbol{F}}$ is the holding power vector of the nonlinear oil film; $\boldsymbol{R}$ is the unbalanced force vector; $\boldsymbol{G}$ is the gravity vector of the system; $\bar{X}$ is the displacement vector of the system.

The form of the system Rayleigh material damping is as following:

$$
\boldsymbol{D}=\alpha \cdot \boldsymbol{M}+\beta \cdot \boldsymbol{K}
$$

where, $\quad \alpha=2\left(\frac{\xi_{2}}{\omega_{2}}-\frac{\xi_{1}}{\omega_{1}}\right) /\left(\frac{1}{\omega_{2}^{2}}-\frac{1}{\omega_{1}^{2}}\right), \quad \beta=2\left(\xi_{2} \omega_{2}-\xi_{1} \omega_{1}\right) /\left(\omega_{2}^{2}-\omega_{1}\right)$

$\xi_{1}, \xi_{2}$-damping parameter, $\omega_{1}, \omega_{2}$ - the first two natural frequency of the system, 
Material damping is usually relative to the calculation results of the rigid support situation. The test system circular frequency and damping parameter are shown in the following table 1.

Table 1 Natural frequency and damping parameters of the system

\begin{tabular}{llllll}
\hline first order & second order & third order & forth order & \multicolumn{2}{l}{ damping parameter } \\
\hline 559.334 & 2454.529 & 6117.442 & $\zeta_{1}=0.05$ & $\zeta_{2}=0.08$ & 559.334 \\
\hline
\end{tabular}

\section{Analysis on bifurcation stability and stability of the eccentric initial phase system}

Accumulated errors such as processing and installation can make eccentric direction of each impeller different easily in practical engineering applications. Therefore, the research content in this section also has a certain engineering value. This section researches on the different eccentric initial phases when the eccentricity of disk b equals $0.10 \mathrm{~mm}$ only.

Through the system stability analysis when $\Delta \varphi$ equals $\pi / 2$ and $\Delta \varphi$ equals $\pi$ previously, it is found that the initial direction of the eccentricity has a great impact on the system stability and it is necessary for more detailed analysis. Fig. 2 shows the bifurcation set in eccentric initial phase difference-speed parameters domain, instability rules described respectively in Cartesian coordinate system and polar coordinate system. Fig. 2(a) shows that set A is the eccentric instability bifurcation set when the eccentricity $b$ equals $0.05 \mathrm{~mm}$, which has been performing for the Hopf bifurcation. Set B(B1, B2) is the eccentric instability bifurcation set when eccentricity b equals $0.05 \mathrm{~mm}$, where B1 is period-doubling bifurcation and B2 is the Hopf bifurcation. It can be seen from the figure, no matter how much the amount of eccentricity is, the instability bifurcation of the system is always symmetric about $\pi$; in addition, with the initial eccentric phase close to $\pi$, the instability speed of the system is also rising, especially the small eccentricity; when the eccentricity is larger, the maximum speed of instability is not the place in $\pi$, but in its sides.

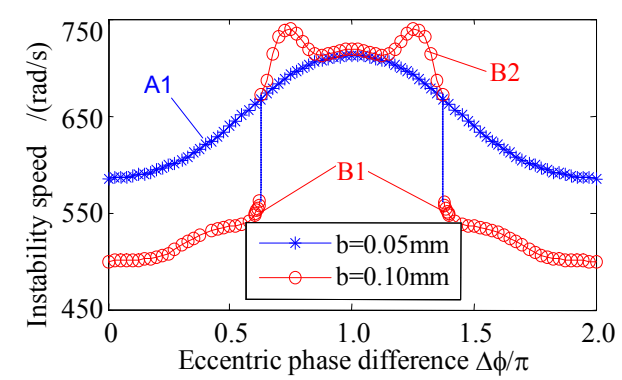

Fig. 2 The bifurcation set in phase difference-rotational speed parameters plane

To verify the correctness of the previous results, the global response bifurcation diagram (Fig. 3) of the system is further calculated when $\Delta \varphi$ equals $\pi$ or $\pi / 2$, from which it can be seen that the period-doubling bifurcation occurs suddenly when $\omega$ equals $536 \mathrm{rad} / \mathrm{s}$ and later turns into Hopf bifurcation, followed by possible chaotic motion or oil whip not discussed here.

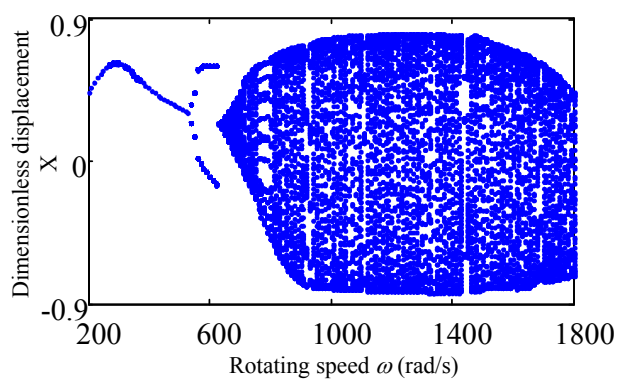

Fig. 3 Bifurcation diagrams of $\mathrm{x}$-direction at $\Delta \varphi=\pi / 2$

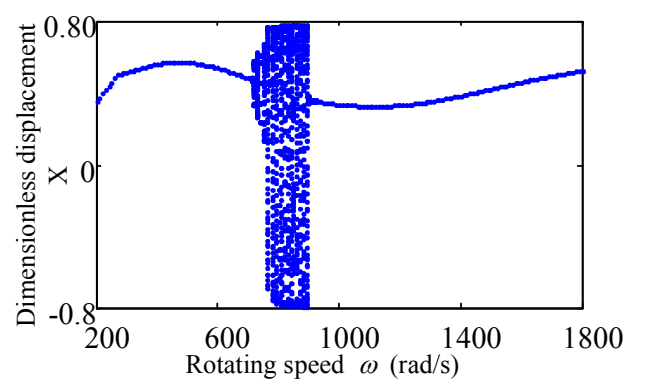

Fig. 4 Bifurcation diagrams of $\mathrm{x}$-direction at $\Delta \varphi=\pi$ 
Fig. 4 is the global response bifurcation diagram of the system when $\Delta \varphi$ equals $\pi$, from which it can be seen that Hopf bifurcation occurs when $\omega=718 \mathrm{rad} / \mathrm{s}$ and later enters co-frequency periodic motion through backward bifurcation.

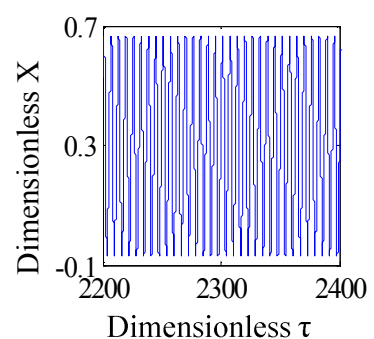

(a) time wave plot

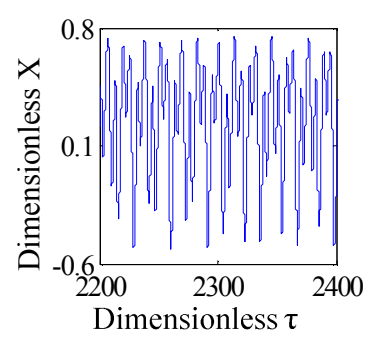

(e) time wave plot

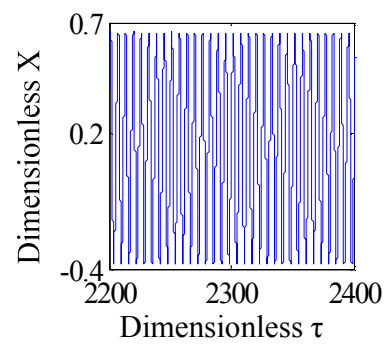

(i) time wave plot

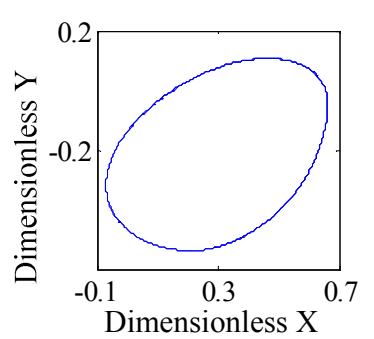

(b) axis orbit

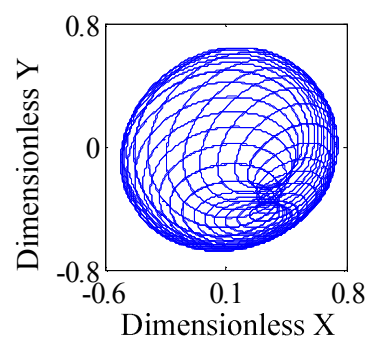

(f) axis orbit

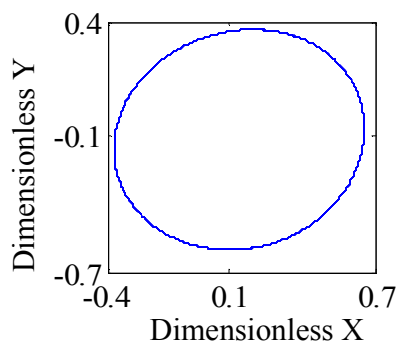

(j) axis orbit

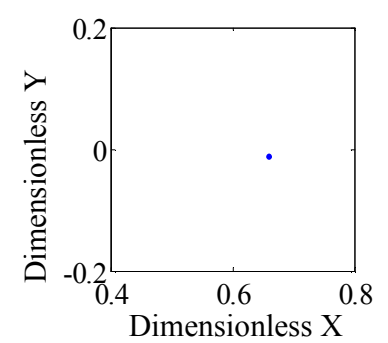

(c) Poincaré map

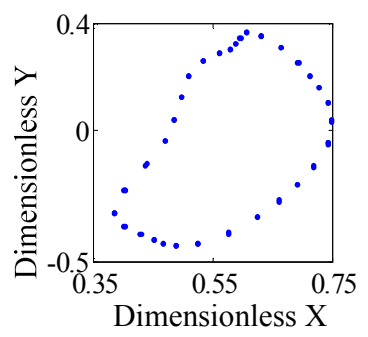

(g) Poincaré map

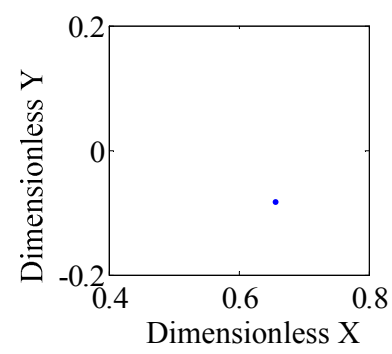

(k) Poincaré map

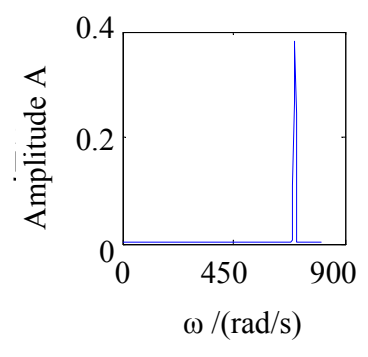

(d) spectrum diagram

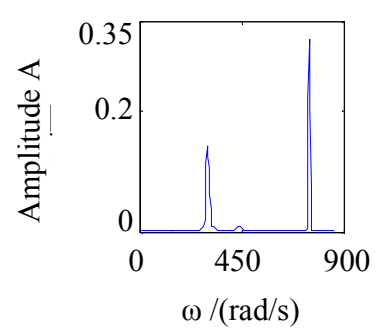

(h) spectrum diagram

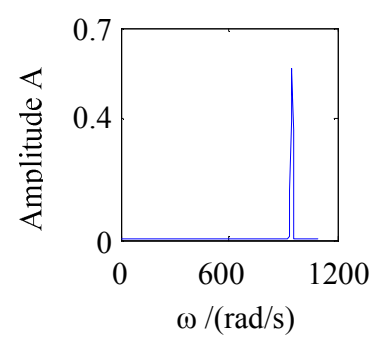

(1) spectrum diagram

Fig. 5 Time wave plot, trajectory, Poincaré maps and amplitude spectra when $\Delta \varphi=\pi \quad \& b=0.10 \mathrm{~mm}$

In order to prove the correctness of the bifurcation and instability speed in front, investigation of the response was did in time and frequency domain only for the case $\Delta \varphi=\pi$ and $b=0.10 \mathrm{~mm}$. As shown in Fig. 5 (a)-(d) are the time wave plot, axis orbit, Poincare section and amplitude spectrum diagram when the initial phase difference $\Delta \varphi=\pi$, rotational speed $\omega=700 \mathrm{rad} / \mathrm{s}$. It can be seen for the result that the system performance for the same frequency response cycle-motion. If the speed of the system was raised further, the response of the system will convert to Hopf bifurcation witch can be seen for Fig. 5 (e)-(h). These figures are time wave plot, axis orbit, Poincare section and amplitude spectrum diagram when rotational speed $\omega=750 \mathrm{rad} / \mathrm{s}$, seen from the amplitude spectrum diagram, a half times rotational frequency displayed in the figure, System performance to moalmost periodic motion. Fig. 5 (i)-(l) are the time wave plot, axis orbit, Poincare section and amplitude spectrum diagram of the system when the initial phase difference $\Delta \varphi=\pi$, rotational speed $\omega=950 \mathrm{rad} / \mathrm{s}$, and the system performance for the same frequency response cycle-motion seen from these results. The results of these figure are consistent with the Fig. 4 and Fig. 2.

\section{Conclusion}

The influence of eccentric initial phase on the dynamic behavior of the double-disc rotor-bearing system is studied by the continuation-shooting algorithm for periodic solution of nonlinear non-autonomous system, and it is found that the instability of co-frequency periodic motion mainly because of period-doubling and Hopf bifurcation. 
The research shows that the initial eccentric has a large impact on the instability speed. The results have shown that the instability speed rises constantly with the eccentric phase difference angle increasing in small eccentricity system. When the two eccentric be in opposite direction, the system reached its maximum instability speed. However, the unstable bifurcation generates mutation phenomenon for large eccentricity system with the eccentric phase difference angle increasing. In summary, the instability speed elevates with difference angle increases. The larger initial phase angle can inhibit system instability partly.

\section{Acknowledgements}

We are grateful to the Fundamental Research Funds for the Central Universities (Grant No. N100303001) and The Research Fund for the Doctoral Program of Higher Education (Grant No. 20100480056) for providing financial support for this work.

\section{References}

[1] R. Brancati, E. Rocca, M. Russo, et al, Journal orbits and their stability for rigid unbalance rotor, ASME Journal of Tribology. 117 (1995) 709-716.

[2] J. Kicinski, R. Drozdowski, P. Materny, The nonlinear analysis of the effect of support construction properties on the dynamic properties of multi-support rotor systems, Journal of Sound And Vibration. 206(1997) 523-539.

[3] N.S. Feng, E.J. Hahn, Vibration analysis of statically indeterminate rotors with hydrodynamic bearing, Trans. ASME Journal of Tribology. 120 (1998) 781-788.

[4] Y.H. Jiao, M. L. Li, Z.B. Chen, Dynamic analysis of rotor-cylindrical bearing system with different oil film force models, Journal of Harbin Institute of Technology. 39(2007) 46-50.

[5] T.S. Zheng, T. Hasebe, Nonlinear dynamic behaviors of a complex rotor-bearing system, Journal of Applied Mechanics. 67(2000) 485-495.

[6] J.P. Jing, G. Meng, Y. Sun, et al. On the nonlinear dynamic behavior of a rotor-bearing system, Journal of Sound and Vibration. 274(2004) 1031-10440.

[7] J.P. Jing, G. Meng, Y. Sun, et al. On the oil-whipping of a rotor-bearing system by a continuum model, Applied Mathematical Modelling. 29 (2005) 461-475.

[8] L.G. Wang, D.Q. Cao, J.L. Wang, et al. Stability and bifurcation of elliptic bearing rotor systems, Journal of Aerospace Power. 23 (2008) 263-269. 